\title{
Two Remarks on Reconstructing Binary Vectors from Their Absorbed Projections
}

\author{
Attila Kuba ${ }^{1}$ and Gerhard J. Woeginger ${ }^{2}$ \\ 1 Department of Image Processing and Computer Graphics, \\ University of Szeged, H-6720, Szeged Árpád tér 2., Hungary \\ kuba@inf.u-szeged.hu \\ 2 Department of Mathematics and Computer Science,
}

TU Eindhoven, P.O. Box 513, 5600 MB Eindhoven, The Netherlands

kgwoegi@win.tue.nl

\begin{abstract}
We prove two small results on the reconstruction of binary matrices from their absorbed projections: (1) If the absorption constant is the positive root of $x^{2}+x-1=0$, then every row is uniquely determined by its left and right projections. (2) If the absorption constant is the root of $x^{4}-x^{3}-x^{2}-x+1=0$ with $0<x<1$, then in general a row is not uniquely determined by its left and right projections.
\end{abstract}

\section{Introduction}

The reconstruction of binary matrices from their row and column sums is a basic problem in discrete tomography, and work on this problem goes back to a seminal paper of Ryser [5] from 1957. Recently, Kuba and Nivat 4 introduced a new discrete tomography model that they call the emission discrete tomography model, EDT for short. In this EDT model, the whole space is filled with some homogeneous partially absorbing material, and the function to be reconstructed represents an object emitting radioactive rays into the surrounding space. Hence, the measurements in EDT are absorbed projections that depend on both, the emitting object and the absorption.

Formally, a picture is a binary $m \times n$ matrix $A=\left(a_{i, j}\right)_{m \times n}$. The entries $a_{i, j}$ are from $\{0,1\}$, and they are sometimes called pixels. Measurements of the picture are taken by sending rays through the material; since the material is partially absorbing, these rays become gradually weaker as they proceed through the material. In 2, 3, 4, the absorption behavior is characterized by the absorption constant

$$
\beta=\frac{1}{2}(-1+\sqrt{5})
$$

where $\beta \approx 0.618$ is the positive root of the equation

$$
\beta^{2}+\beta=1
$$


Then the absorbed left projection $\mathrm{ALP}_{i}$ of row $i$ is defined as

$$
\mathrm{ALP}_{i}=\sum_{j=1}^{n} a_{i, j} \cdot \beta^{j}
$$

and the absorbed right projection $\mathrm{ARP}_{i}$ of row $i$ is defined as

$$
\mathrm{ARP}_{i}=\sum_{j=1}^{n} a_{i, j} \cdot \beta^{n+1-j} .
$$

Intuitively speaking, in the left projection the ray enters the row from the left hand side. As the ray passes through the pixels at the beginning of the row, it is still strong, and provides precise information. Then the ray becomes gradually absorbed, and the factors $\beta^{j}$ in (3) encode this absorption. In the right projection, the ray enters the row symmetrically from the right hand side, and this leads to the symmetric formula given in (4). Absorbed upward and downward projections of columns are defined analogously.

The papers by Kuba and Nivat [4, Balogh et al. [2], and Kuba et al. 3] discuss a variety of algorithmical and combinatorial questions around reconstruction problems with absorbed projections. Noteworthy, all these papers only study the cases with absorbed left projections of rows (but without right projections) and with absorbed upward projections of columns (but without downward projections). Barcucci et al. [1] proved that the left and right absorbed projections determine uniquely the binary matrix if the absorbtion coefficient is $\mu=\log ((1+\sqrt{5}) / 2)$ and they gave an algorithm for the reconstruction.

In this note, we will show that the corresponding problems where the left and right absorbed row projections are known simultaneously are rigid and highly constrained. Our combinatorial proof is different from the proof given by Barcucci et al. 1] in the sense that it does not use the concenpt of switching component.

Theorem 1. In the EDT model with absorption constant $\beta=\frac{1}{2}(-1+\sqrt{5})$, the left and the right absorbed projections $A L P_{i}$ and $A R P_{i}$ determine the row $r_{i}$ uniquely.

Up to now, we have only considered the basic EDT model with absorption constant $\beta=\frac{1}{2}(-1+\sqrt{5}$ ) (which arguably is the simplest non-trivial EDT model). Interestingly, the statement in Theorem 1 does not generalize to arbitrary values of the absorption constant:

Theorem 2. Consider the EDT model where the absorption constant

$$
\gamma=\frac{1}{4}(\sqrt{13}+1-\sqrt{2 \sqrt{13}-2}) \approx 0.581
$$

is the unique real root of $\gamma^{4}-\gamma^{3}-\gamma^{2}-\gamma+1=0$ that satisfies $0<\gamma<1$. Then the left and the right absorbed projections $A L P_{i}$ and $A R P_{i}$ in general do not determine a row $r_{i}$ uniquely. 


\section{Proof of the Unique Reconstruction Result}

In this section we prove Theorem 1. Throughout, let $\beta=\frac{1}{2}(-1+\sqrt{5})$ be defined as in (2), and let $\alpha=\frac{1}{2}(1+\sqrt{5})$ denote the positive root of $\alpha^{2}=\alpha+1$. Note that $\alpha=1 / \beta$. Furthermore, we define the Fibonacci numbers as usual by

$$
F_{-3}=-1 \quad F_{-2}=1 \quad F_{-1}=0 \quad F_{0}=1 \quad F_{1}=1
$$

and by $F_{n}=F_{n-1}+F_{n-2}$ for all $n \geq 2$.

Proposition 1. The following statements on the Fibonacci numbers are wellknown:

(i) $\alpha^{i}=F_{i-1} \alpha+F_{i-2}$ for all $i \geq 0$.

(ii) $\beta^{i}=(-1)^{i-1} F_{i-1} \beta+(-1)^{i-2} F_{i-2}$ for all $i \geq 0$.

(iii) $F_{i+2}-1=F_{i}+F_{i-1}+\cdots+F_{1}+F_{0}$ for all $i \geq 0$.

Proof. By induction on $i$.

Proposition 2. Let $x_{i} \in\{-1,0,1\}$ for $i \geq 0$. Then the following statements hold true:

(i) If $\sum_{i=0}^{\ell} F_{2 i} \cdot x_{2 i}=0$, then $x_{2 i}=0$ for $i=0, \ldots, \ell$.

(ii) If $\sum_{i=0}^{\ell} F_{2 i+1} \cdot x_{2 i+1}=0$, then $x_{2 i+1}=0$ for $i=0, \ldots, \ell$.

Proof. Proof of statement (i). Suppose for the sake of contradiction that $x_{2 i} \neq 0$ for some $i$, and consider the largest index $k$ with $x_{2 k} \neq 0$. Without loss of generality, we may assume that $x_{2 k}=-1$ (and otherwise, we multiply all $x_{2 k}$ by -1$)$. Then $\sum_{i=1}^{k-1} F_{2 i} \cdot x_{2 i}-F_{2 k}=0$. This leads to

$$
\begin{aligned}
F_{2 k} & =\sum_{i=0}^{k-1} F_{2 i} \cdot x_{2 i} \leq \sum_{i=0}^{k-1} F_{2 i} \\
& =\sum_{i=0}^{k-1} F_{2 i-2}+F_{2 i-1}=\sum_{i=-2}^{2 k-3} F_{i}<1+\sum_{i=0}^{2 k-2} F_{i} .
\end{aligned}
$$

This blatantly contradicts statement (iii) in Proposition [1, and proves statement (i). Statement (ii) can be handled similarly: Let $k$ be the largest index $k$ with $x_{2 k+1} \neq 0$, and assume that $x_{2 k+1}=-1$. Then

$$
\begin{aligned}
F_{2 k+1} & =\sum_{i=0}^{k-1} F_{2 i+1} \cdot x_{2 i+1} \leq \sum_{i=0}^{k-1} F_{2 i+1} \\
& =\sum_{i=0}^{k-1} F_{2 i-1}+F_{2 i}=\sum_{i=-1}^{2 k-2} F_{i}=\sum_{i=0}^{2 k-2} F_{i} .
\end{aligned}
$$

This contradicts Proposition 1).(iii) and completes the proof of statement (ii). 
Now consider two rows $r_{1}=\left\langle a_{1}, \ldots, a_{n}\right\rangle$ and $r_{2}=\left\langle b_{1}, \ldots, b_{n}\right\rangle$ with pixels $a_{i}, b_{i} \in\{0,1\}$ in some binary picture. We assume that the left projections of these two rows are identical, i.e.,

$$
\sum_{i=1}^{n} a_{i} \beta^{i}=\sum_{i=1}^{n} b_{i} \beta^{i}
$$

and that also their right projections are identical, i.e.,

$$
\sum_{i=1}^{n} a_{i} \beta^{n+1-i}=\sum_{i=1}^{n} b_{i} \beta^{n+1-i} .
$$

For $i=1, \ldots, n$ we define $c_{i}=a_{i}-b_{i}$ with $c_{i} \in\{-1,0,+1\}$. Furthermore, we introduce the four auxiliary quantities $A=\sum_{i=1}^{n} F_{i-2} c_{i}, B=\sum_{i=1}^{n} F_{i-3} c_{i}$, $C=\sum_{i=1}^{n}(-1)^{i-2} F_{i-2} c_{i}$, and $D=\sum_{i=1}^{n}(-1)^{i-3} F_{i-3} c_{i}$.

Now Equation (7) can be written as $\sum_{i=1}^{n} \beta^{i} c_{i}=0$. By using statements (i) and (ii) in Proposition 1, this leads to

$$
0=\sum_{i=1}^{n} \beta^{i} c_{i}=\sum_{i=1}^{n}\left((-1)^{i-1} F_{i-1} \beta+(-1)^{i-2} F_{i-2}\right) c_{i}=\beta(D-C)+C .
$$

Similarly, Equation (8) can be written as $\sum_{i=1}^{n} \alpha^{i} c_{i}=0$ and yields

$$
0=\sum_{i=1}^{n} \alpha^{i} c_{i}=\sum_{i=1}^{n}\left(F_{i-1} \alpha+F_{i-2}\right) c_{i}=\alpha(A+B)+A .
$$

Since $\alpha$ and $\beta$ are irrational numbers, whereas $D-C, C, A+B, A$ are integers, the Equations (9) and (10) imply $D-C=0, C=0, A+B=0$, and $A=0$. Hence, $A=B=C=D=0$ holds. From this we derive

$$
0=A+C=\sum_{i=1}^{n}\left(1+(-1)^{i-2}\right) F_{i-2} c_{i}=2 \sum_{i=1}^{\lfloor n / 2\rfloor} F_{2 i-2} c_{2 i} .
$$

Equation (11) together with Proposition 2,(i) now yields that all $c_{i}$ with an even index are 0 . Furthermore, we derive for the odd indices that $A-C=0$ and therefore

$$
0=\sum_{i=1}^{n}\left(1-(-1)^{i-2}\right) F_{i-2} c_{i}=2 \sum_{i=1}^{\lceil n / 2\rceil} F_{2 i-3} c_{2 i-1}=2 \sum_{i=2}^{\lceil n / 2\rceil} F_{2 i-3} c_{2 i-1} .
$$

Here we used in the final step that $F_{-1}=0$. Equation (12) together with Proposition 2, (ii) now yields that all $c_{i}$ with an odd index $i \geq 3$ are 0 . What about $c_{1}$ ? The value of $B=\sum_{i=1}^{n} F_{i-3} c_{i}$ boils down to $B=F_{-2} c_{1}=c_{1}$, and together with $B=0$ this implies that also $c_{1}=0$. Summarizing, $c_{i}=0$ must hold for all $i=1, \ldots, n$. This implies $a_{i}=b_{i}$ for all $i=1, \ldots, n$. Hence, the two rows $r_{1}$ and $r_{2}$ must be identical, and any row is uniquely determined by its left and right absorbed projections. This completes the proof of Theorem 1 .

Remark 1. About absorption values for which any binary matrix is uniquely determined by its absorbed row sums see Section 6 in $[3]$. 


\section{Proof of the Non-unique Reconstruction Result}

In this section we prove Theorem 2. Hence, consider an absorption constant $\gamma$ with $0<\gamma<1$ that is a root of $1+\gamma^{4}=\gamma+\gamma^{2}+\gamma^{3}$ as defined in (5). Consider a $2 \times 10$ matrix with two rows $r_{1}=\langle 1,0,0,0,1,1,0,0,0,1\rangle$ and $r_{2}=$ $\langle 0,1,1,1,0,0,1,1,1,0\rangle$. Then

$$
\begin{aligned}
\operatorname{ALP}_{1} & =\gamma+\gamma^{5}+\gamma^{6}+\gamma^{10}=\left(\gamma+\gamma^{6}\right)\left(1+\gamma^{4}\right) \\
& =\left(\gamma+\gamma^{6}\right)\left(\gamma+\gamma^{2}+\gamma^{3}\right) \\
& =\gamma^{2}+\gamma^{3}+\gamma^{4}+\gamma^{7}+\gamma^{8}+\gamma^{9}=\mathrm{ALP}_{2} .
\end{aligned}
$$

Since rows $r_{1}$ and $r_{2}$ both are left-right symmetric, this chain of equations also yields $\mathrm{ARP}_{1}=\mathrm{ARP}_{2}$. Summarizing, these two rows both have the same absorbed left projection and both have the same absorbed right projection. Generally, it is not possible to uniquely reconstruct a row from its two projections. This proves Theorem 2 ,

Remark 2. Of course, the left and right absorbed projections determine the binary vector uniquely if its size is small enough, that is, if $n \leq 4$. The explanation is that a binary vector is non-uniquely determined by its left and right absorbed projections if and only if the equation $1+\gamma^{4}=\gamma+\gamma^{2}+\gamma^{3}$ can be applied in the description of its value. This equation requires at least 5 binary digits.

\section{Acknowledgment}

This work was supported by the NSF Grant DMS 0306215.

\section{References}

1. Barcucci, E., Frosini, A., Rinaldi, S.: Reconstruction of discrete sets from two absorbed projections: an algorithm. Electronic Notes on Discrete Mathematics 12 (2003)

2. Balogh, E., Kuba, A., Del Lungo, A., Nivat, M.: Reconstruction of binary matrices from absorbed projections. In Proceedings of the 10th International Conference on Discrete Geometry for Computer Imagery (DGCI'2002), Springer LNCS 2301 (2002) 392-403

3. Kuba, A., Nagy, A., Balogh, E.: Reconstuction of hv-convex binary matrices from their absorbed projections. Discrete Applied Mathematics 139 (2004) 137-148

4. Kuba, A., Nivat, M.: Reconstruction of discrete sets with absorption. Linear Algebra and Applications 339 (2001) 171-194

5. Ryser, H.J.: Combinatorial properties of matrices of zeros and ones. Canadian Journal of Mathematics 9 (1957) 371-377 